This is the pre-peer reviewed version of the following article:

Sledzinska M., Graczykowski B., Maire J., Chavez-Angel E., Sotomayor-Torres C.M., Alzina F.. 2D Phononic Crystals:

Progress and Prospects in Hypersound and Thermal Transport Engineering. Advanced Functional Materials, (2019). .

1904434: - . 10.1002/adfm.201904434,

which has been published in final form at https://dx.doi.org/10.1002/adfm.201904434. This article may be used for non-commercial purposes in accordance with Wiley Terms and Conditions for Use of Self-Archived Versions. 


\section{Two-Dimensional Phononic Crystals: Progress and Prospects in Hypersound and Thermal Transport Engineering}

Marianna Sledzinska, Bartlomiej Graczykowski, Jeremie Maire, Emigdio Chavez-Angel, Clivia M. Sotomayor-Torres*, and Francesc Alzina

Dr. Marianna Sledzinska

Catalan Institute of Nanoscience and Nanotechnology (ICN2), CSIC and BIST, Campus UAB, Bellaterra, 08193 Barcelona, Spain.

Dr. Bartlomiej Graczykowski

Faculty of Physics, Adam Mickiewicz University, Umultowska 85, 61614 Poznan, Poland.

Max Planck Institute for Polymer Research, Ackermannweg 10, 55218 Mainz, Germany.

Dr. Jeremie Maire, Dr. Emigdio Chavez-Angel

Catalan Institute of Nanoscience and Nanotechnology (ICN2), CSIC and BIST, Campus UAB, Bellaterra, 08193 Barcelona, Spain.

Prof. Dr. Clivia M. Sotomayor-Torres

Catalan Institute of Nanoscience and Nanotechnology (ICN2), CSIC and BIST, Campus UAB, Bellaterra, 08193 Barcelona, Spain.

ICREA, Passeig Lluis Companys 23, 08010 Barcelona, Spain.

E-mail: clivia.sotomayor@icn2.cat

Dr. Francesc Alzina

Catalan Institute of Nanoscience and Nanotechnology (ICN2), CSIC and BIST, Campus UAB, Bellaterra, 08193 Barcelona, Spain.

Keywords

phononics, 2D phononic crystals, hypersound, elastic waves, nanoscale thermal transport

\section{Abstract}

The central concept in phononics is the tuning of the phonon dispersion relation, or phonon engineering, which provides a means of controlling related properties such as group velocity or phonon interactions and, therefore, phonon propagation, in a wide range of frequencies depending on the geometries and sizes of the materials. Phononics exploits present state of the art in nanofabrication to tailor dispersion relations in the range of $\mathrm{GHz}$ for the control of elastic waves/phonons propagation with applications towards new information technology concepts with phonons as state variable. Moreover, phonons provide an adaptable approach for supporting a coherent coupling between different state variables, and the development of 
nanoscale optomechanical systems during the last decade attests this prospect. The most extended approach to manipulate the phonon dispersion relation is introducing an artificial periodic modulation of the elastic properties, which is referred as phononic crystal (PnC). In this progress report, we focus in recent experimental achievements in the fabrication and application of two-dimensional (2D) PnCs enabling the modification of the dispersion relation of surface and membrane modes, and presenting phononic band gaps, waveguiding and confinement in the hypersonic regime. Furthermore, these artificial materials offer the potential of modifying and controlling the heat flow to enable new schemes in thermal management.

\section{Introduction}

The propagation of sound always happens in a medium, either solid, liquid or gas, and is described as propagating waves at a velocity that depends on the elastic properties of the material (stiffness and mass density). The importance of sound in everyday life, industry and science goes far beyond our natural perception and common understanding. For instance, at low frequencies below our hearing threshold, there are infrasound of great importance in seismography and animal communication. On the other hand, above hearing threshold, we find broad spectra of frequencies, which are crucial in medical imagining (ultrasounds), signal processing (hypersounds) and thermal or heat transport. Sound, in addition to its wave-like nature can be described by means of (quasi) particles, quanta of vibrational energy, i.e., phonons. In analogy to photons and electrons, phonons are carriers of energy, momentum and information. Phonons, as an expression of atomic motion, are highly interactive with all excitations present in a material ${ }^{[1,2]}$. As a consequence, the nature of phonons allows for, at least, two pathways of their engineering: trivial absence of medium or subtle manipulation of the condensed matter utilizing both wave- and particle-like description of phonons. 
The central concept in phononics is the tuning of the phonon dispersion relation or phonon engineering, which provides a means of controlling related properties such as group velocity or phonon interactions and, as a result, of phonon propagation in a wide range of frequencies depending on the geometries and sizes of the materials. ${ }^{[3]}$ Phononics exploits present state of the art in nanofabrication to tailor dispersion relations in the hypersonic range for the control of phonon propagation as a way to circumvent the limitations in present applications, as well as a road towards new information technology concepts with phonons as state variable. As classical waves, the behaviour of the elastic waves is determined by the interfaces and, more specifically, by the mismatch between the elastic properties of the two phases that the boundary separates. Therefore, they are at the origin of the positive impact of boundary scattering, which is the basis of waveguiding and of the surface and interface waves. ${ }^{[4]}$ In plates and layered materials, for example, this leads to multiple non-linear spatial dispersive phonon modes, which propagate in-plane, where the plate or layer thickness acts as the tuning parameter ${ }^{[5-8]}$ Further control of the phonon dispersion relation is attained by a periodic modulation of the elastic properties in PnCs. ${ }^{[9,10]}$ The interest in PnCs has often been related with the occurrence of frequency band gaps, which appear as a consequence of Bragg scattering when the phonon wavelength has a definite relation to the periodicity, but also to the resulting modification of the dispersion relation. As long as the introduced artificial periodicity has a characteristic period larger than the atomic lattice, the continuum approach is still valid to describe such materials. Indirect and direct measurements of the modified hypersonic phonon dispersion has been performed in surface and plate two-dimensional (2D) PnCs by means of electro-acoustic platforms ${ }^{[11]}$ and Brillouin light scattering (BLS). ${ }^{[12-14]}$

Basic research and device applications based on the interaction between elastic and optical waves are also benefiting from the studies of PnCs towards wave propagation manipulation. The confinement of both optical and elastic fields in the same reduced volumes of simultaneous 2D photonic and phononic crystal based resonators allows for integrated 
acousto-optic devices and has already brought these optomechanical structures to impact basic research of different disciplines. ${ }^{[15]}$

Finally, phononics is re-examining the role of phonons as heat carriers, and how thermal properties can be tuned by materials engineering at the nanoscale. In this context, 2D PnCs structures with characteristic sizes exhibiting wave-like behaviour in the hypersound regime were found to exhibit reduced in-plane thermal conductivity $(\kappa)$ at room temperature and beyond. ${ }^{[16,17]}$ In the latter conditions, the thermal conductivity results from the cumulative contribution of phonons characterized by a broad range of wavelengths despite the predominance of short-wavelength phonons. Therefore, the periodicity itself is not expected to impact the thermal transport, and the reduction of the in-plane thermal conductivity is mainly directed by the diffusive scattering of phonons at the boundaries of the $2 \mathrm{D} \mathrm{PnC}$. At low temperatures, however, the wavelength of the phonons is large enough so that their spatial dispersion is strongly affected by characteristic sizes of the order of the micrometer and sub-micrometer. ${ }^{[18,19]}$

The determination of the thermal conductance and the further extraction of thermal properties, such as the thermal conductivity of a given sample, requires the measurement of the temperature difference in the heat flow direction. The present electrical techniques employed to obtain the thermal conductivity are based on the temperature dependence of an electrically resistive element ${ }^{[20]}$ or on the tunnel current in a normal-metal-insulatorsuperconductor junction. ${ }^{[21]}$ However, two major drawbacks are the need for complex fabrication and for a well-controlled thermal resistance introduced by the heater-thermometer pair. Therefore, non-contact optothermal methods such as, for example, time-domain thermoreflectance ${ }^{[22]}$ and Raman thermometry ${ }^{[23]}$ have been developed and are currently extensively available. While in time-domain thermoreflectance the changes in the refractive index of a transducer material is used as temperature sensor, in Raman thermometry, the 
temperature dependence of the Raman scattering by phonons is used to obtain the temperature of the sample.

In this progress report we present some representative examples of the recent developments achieved in the topics of 2D PnCs for the control of propagating elastic waves in the frequency regime of hypersound and heat transport by phonons. This research is anchored in the development of novel methods to produce nanostructures pushing towards the limits of nanofabrication. We have organized this report by summarizing the state of the art of fabrication techniques being used to fabricate structures with characteristic sizes in the submicrometer range and others being developed to reduce feature size well in the nanoscale. Then, the key schemes reported to change the dispersion relation of elastic waves in surfaces and plates by using 2D PnCs structures are presented, together with their experimental realization with operating frequencies in the $\mathrm{GHz}$ range. Next, we summarize the recent progress in the study of heat transport by phonons in these structures, pointing out the different effects or regimes, coherent and incoherent, depending on the feature size and the measuring temperature. Finally, we provide some of the directions that the research related to these systems progress incorporating new concepts for controlling elastic waves propagation and heat transport, and the prospect of assembling hybrid electro-opto-mechanical platforms and circuits, or functionalities analogous to those found in electronics enabling thermal circuits.

\section{Fabrication Techniques}

The feature size of 2D PnCs in the hypersonic range, typically of about few hundreds of nanometres, is easily accessible with standard fabrication techniques, which can be divided into the 'top-down' and 'bottom-up' methods. In the top-down method a bulk sample is structured in order to obtain small scale features, while the bottom-up approach starts at the molecular level assembling the building blocks into larger entities. In this section we will first 
discuss the top-down methods for solid state PnCs, followed by the bottom-up methods applied to colloidal crystals. Finally, an outlook on the fabrication challenges is provided.

Electron beam lithography (EBL), a lithographic technique widely employed to define nanoscale features is used to write the designed pattern on a sample previously spin-coated with a resist, which is exposed to a high-energy electron beam. Depending on the nature of the resist, the electron beam can either polymerize (negative) or depolymerize (positive) it. A development step removes the unpolymerized resist and exposes the pattern (Figure 1 (a)). Then, reactive ion etching (RIE) can be used to transfer the pattern to the substrate ${ }^{[24]}$ or metal evaporation and lift off can be used to fabricate an array of metallic features, such as lines or pillars on the samples surface, forming a surface PnC (Figure 1 (b) and (c)). ${ }^{[12,25-27]}$

The development of the silicon-on-insulator (SOI) technology has enabled advances in the field of nanophononics by providing high quality single-crystal silicon layers with thickness down to a few tens of nanometers, ${ }^{[28]}$ which can be employed in order to fabricate free-standing PnC membranes (Figure 1 (d)). ${ }^{[29]}$ After patterning the device silicon layer, the membrane contour is defined in a second EBL and RIE step, and finally the membrane is suspended by etching the buried $\mathrm{SiO}_{2}$ layer using $\mathrm{HF}$ acid or buffered $\mathrm{HF}$. In order to avoid the collapse of the membranes critical point drying is employed ${ }^{[14,30,31]}$ or alternatively vapor HF can be used order to reduce the surface tension during the etching. ${ }^{[16,32]}$

The main limitation of the SOI-based, under-etched PnCs is the structure size, typically a few tens of micrometers. Larger structures tend to collapse due to the internal strain of the top Si layer. In order to fabricate large area membranes a full back-etching process has to be performed, i.e., both the handle silicon substrate and the $\mathrm{SiO}_{2}$ layers have to be removed. ${ }^{[33,34]}$ We have shown that such free-standing membranes with thickness down to $100 \mathrm{~nm}$ can be further used to fabricate both solid-air and solid-solid PnCs using standard EBL process. ${ }^{[17,35-37]}$ 
Finally, other techniques have been employed to fabricate 2D PnCs, such as focused ion beam (FIB) milling, which has the advantage of direct substrate patterning, with no need of further pattern transfer. For instance, FIB milling was used to realize a PnC fabricated in a free-standing silicon membrane ${ }^{[38]}$ and showing frequency band gaps above $25 \mathrm{GHz}$. FIB milling can be used for other materials, especially those difficult to etch with dry etching techniques, such as $\mathrm{LiNbO}_{3}{ }^{[39]}$. Still, the main issue of this technique is the long exposure time, control of damage and the fact that the holes are normally conical in shape, with the top diameter larger than the bottom one.

For $\mathrm{LiNbO}_{3}$ another nanostructuring technique was proposed, based on domain inversion process induced by high power laser irradiation. ${ }^{[40,41]}$ A patterned chromium mask is deposited on the sample surface before irradiation with a wide diameter beam of intense visible laser light. The regions patterned with chromium are domain inverted while the uncoated regions are not affected by the irradiation. The domain inversion is caused due to the local heating of the crystal and a diffusion process in which $\mathrm{Cr}$ reacts with $\mathrm{LiNbO}_{3}$ oxygen ions, forming $\mathrm{Cr}_{2} \mathrm{O}_{3}$. In a last step, the original domain is removed using wet etching with $\mathrm{HF}$ acid.

2D colloidal crystals on a surface are the most prominent example of bottom-up structures in phononics, offering the possibility of tailoring the length scales, order or porosity $^{[42]}$ (Figure 1 (e)). Colloidal crystals are made of micro- or nanoparticles, assembled into highly ordered lattices. There is a variety of methods for self-assembly of the colloidal crystals, one of them being liquid interface-mediated methods, such as the Langmuir-Blodgett ${ }^{[43]}$ process or a direct assembly process at the air-water interface ${ }^{[44]}$. In this method the particles are trapped at the liquid-air or liquid-liquid interface forming a crystal, which is easy to transfer to a solid substrate.

Modifying the dispersion relation of the high frequency (THz) phonons (see Section 4) poses the major fabrication challenge of reducing the PnCs characteristic sizes in the sub-10 
$\mathrm{nm}$. One of the techniques proposed to achieve sub-10 $\mathrm{nm}$ size features relies on the combination of top-down and bottom-up procedures, namely block-copolymers selfassembly $^{[45]}$. Self-assembled BCPs form a periodic or quasi-periodic array of pores, which can then be transferred to silicon (Figure 1 (f)). Free-standing porous membranes with thickness of $100 \mathrm{~nm}$ and features with neck size down to $16 \mathrm{~nm}^{[46]}$ have been reported, even though the long-range order of the BCP self-assembly remains a challenge. ${ }^{[47]}$

Another nanofabrication issue is the disorder and roughness of the patterned features. The effect of roughness on phonon coherence is of interest to the phononics community (see Section 4 and 5) and it is expected that its role will increase with decreasing the characteristic size of PnCs. While the SOI silicon device layer has a surface roughness of typically below 1 $\mathrm{nm}$, the features, such as holes, present considerable roughness, about $2.5-5 \mathrm{~nm}{ }^{[35,48]}$ which depends on the quality of both, lithography and pattern transfer. In order to reduce the silicon surface and sidewall roughness, partial oxidation can be performed. ${ }^{[49]}$ The rate of oxidation is well documented and can be controlled with the temperature, making it a reliable strategy for reducing roughness, which is commonly applied in silicon nanophotonics. Consecutive thermal oxidation and an oxide removal process applied to free-standing photonic crystal cavities were shown to improve the quality factor of the cavities. ${ }^{[50]}$ Silicon surfaces can also be repaired by a series of chemical treatments, such as repeated chemical oxidation in piranha (H2SO4/H2O2) and HF oxide stripping. ${ }^{[51]}$ These techniques can find a direct application in the fabrication $\mathrm{PnCs},{ }^{[52]}$ leading to major control of the surface and sidewall roughness.

\section{Surface and membrane hypersonic 2D PnCs.}

In this section we focus on recent experimental research on 2D PnCs controlling the propagation of spatially confined hypersonic $(\mathrm{GHz})$ elastic waves/phonons. The advances in related PnCs theoretical studies with different geometries and in different spectral regimes can be found elsewhere ${ }^{[53-56]}$. Applying the scaling principle of PnCs one can adjust their operating frequency up to the GHz regime by reducing the feature size to the sub-micrometer 
range. Well-developed micro- and nano-fabrication of hypersonic PnCs makes them highly attractive for applications in wireless communication, ${ }^{[57,58]}$ thermal transport management ${ }^{[17,19,59]}$ and enhanced phonon-photon interaction in optomechanics. ${ }^{[60,61]}$

Arrangement of PnCs into surface-like structures enables well-developed generation and detection of high frequency signals. Furthermore, confining the elastic field minimizes losses. Such 2D PnCs have been realized on free surfaces of homogenous or layered media or on thin membranes to gain a control over surface acoustic waves (SAWs) $\mathrm{PnCs}^{[12,25-27,41,62-65]}$ or Lamb plate waves (membrane PnCs), ${ }^{[14,35,36,66,67]}$ respectively. In the following we present different strategies for the modification of the phonon dispersion in PnCs and show resulting phenomena from the viewpoint of the experimental techniques employed for their investigation.

Nowadays, it is hard to overestimate the importance of SAWs in miniaturized devices for $\mathrm{GHz}$ signal such as filters, multi- and demultiplexers or delay lines. ${ }^{[57]}$ The concept of manipulating the SAWs dispersion by means of artificial 2D periodic structure was first proposed by Tanaka and Tamura ${ }^{[62]}$. To date the main strategies using PnCs for the modification of SAWs dispersion are based on the introduction of (i) periodically arranged scatterers, (ii) local resonances and (iii) contact resonances (Figure 2 (a)). The first scheme, namely, the periodic repetition of a surface motif, introduces features typical of PnCs such as non-linear band structure, zone folding and Bragg band gap opening. ${ }^{[53]}$ These effects were reported for Rayleigh-like SAWs in PnCs made out of square lattices of holes or inclusions in Si measured by means of time-resolved imaging ${ }^{[54,68]}$ and BLS. ${ }^{[12,13]}$ Furthermore, holey piezoelectric SAW PnCs forming an acoustic cavity for electro-acoustically generated elastic waves were utilized to realize a one-port Love SAW resonator operating in the $\mathrm{GHz}^{[69]}$. The second strategy consists of PnCs formed by a periodic array of local resonators on a free surface, known also as pillar-based PnCs, ${ }^{[25-27,41,53,63-65,70]}$ which offer several advantages over holey or inclusion-based PnCs. Foremost, pillars can work both as periodic scatterers, 
resulting in Bragg band gaps, and as local resonators, introducing narrow sub-wavelength band gaps. ${ }^{[39,53,67]}$ For the latter the forbidden range of frequencies is robust to PnC period or imperfections and can be tailored by the material and geometry of the pillars ${ }^{[35]}$ in order to gain control over the band gap spectral position and width. ${ }^{[53,70]}$ Hypersonic vibrational properties of such systems were investigated by ultrafast time-domain pump-probe techniques $^{[26,63,64]}$ to unravel the coupling of the local resonances with SAWs and with the bulk modes of the substrate, dispersion, lifetime and thermomechanics. Going further, frequency-domain BLS proved structure-induced zone folding and formation of $\mathrm{GHz}$ Bragg and local resonance acoustic band-gaps for thermally populated SAWs pillar-based PnCs made out of $\mathrm{Si}$ or $\mathrm{LiNbO}_{3}{ }^{[25,27,41,65]}$ A similar type of $\mathrm{PnC}$ was recently utilized for the first experimental realization of a Si-compatible $\mathrm{GHz}$ filter made out in an all-dielectric platform $^{[70]}$ The third strategy enabling the modification of SAWs dispersion is based on the nanoparticle-substrate contact resonance. This effect was first investigated in the sub-GHz regime by the pump-probe transient grating (TG) technique ${ }^{[71,72]}$ and time-domain imaging ${ }^{[73]}$ in PnCs made out of self-assembled silica microspheres adhered to an aluminium-coated glass substrate. Recent follow up of the TG study has revealed additional features in the $\mathrm{GHz}$ range such as contact-induced splitting of the vibrational modes of nanoparticles and the interaction of these modes with Rayleigh SAWs ${ }^{[74]}$. Theoretically, this type of SAW PnCs offers all three schemes for bang gap opening, whereas the period and local resonances can be adjusted by the size and material of nanoparticles, contact resonances can be tailored by the particle-substrate adhesion by means of surface physics or chemistry. ${ }^{[4]}$

Despite the advantages of SAW PnCs, there is a drawback resulting from the elastic field leakage into bulk through bulk waves and pseudo-SAWs. ${ }^{[62]}$ Furthermore, to increase the operational frequency requires a proportional reduction in feature size ${ }^{[57]}$ or searching for systems with low-loss supersonic surface waves. ${ }^{[75,76]}$ To overcome this issue one can use membranes as a substrate for PnCs following all three schemes presented in this section. In 
comparison to SAWs, elastic Lamb waves in membranes form a discrete set of dispersive modes giving access to higher frequencies without losses into the substrate. ${ }^{[53,77]}$ Nanofabricated pillar-based or holey membrane PnCs were recently investigated by means of micro-BLS ${ }^{[14,35]}$, Asynchronous Optical Sampling (ASOPS) ${ }^{[36]}$ from the perspective of GHz phonon dynamics, and by various techniques to study phononic thermal transport. ${ }^{[17-19,59,78]}$ Research presented in Ref. ${ }^{[14]}$ considers two-dimensional Si PnCs membrane combining different strategies, such as 1D spatial confinement, periodic modulation, and local resonances, all of which affect the dispersion of hypersonic waves/phonons. Results from BLS (see Figure 2 (b) and (c)) have provided direct experimental evidence on the modification of the dispersion relation of thermally activated phonons up to tens of GHz. That cut-off frequency was attributed to the vanishing of wave-like (coherent) behaviour of phonons at higher frequencies due to the increased role of the $\mathrm{PnC}$ imperfections such as surface roughness and PnC lattice disorder. In particular, the square lattice of holes introduced band folding and splitting, satisfying the Bragg condition, as well as anti-crossing between related branches. Moreover a pseudo-band gap for symmetric modes and a full band gap along a high symmetry direction were observed. In the pillar-based PnC a square lattice of heavy $\mathrm{Au}$ pillars introduced strong modification of the phonon dispersion with an overall frequency downshift of the branches with respect to those of a bare membrane. The hybridization between local resonances in pillars and modes of the underlying membrane resulted in an anti-crossing between the non-dispersive branches of the local resonances and the bands of the propagating modes in the substrate. This can lead to sub-wavelength band gaps, immune to PnC lattice imperfections, which can be tuned by changing the characteristic sizes of the pillars.

The follow up of this investigation performed by means of ASOPS ${ }^{[36]}$ showed that PnC lattice disorder and surface roughness of holes drastically changed the hypersonic phonon spectrum resulting in the suppression of wave-like effects. As a consequence the 
system became an effective medium with no impact on phononic thermal transport at room temperature. Nevertheless, a controllable disarrangement introduced into 2D lattice of PnC gives a prospect of new features such as, bang-gap broadening or Anderson localization.

Membrane-based PnCs offer new features in comparison to SAW PnCs, albeit with a more complex fabrication. The latter can be reduced by use of already mentioned selfassembled systems of micro- and nanoparticles providing low-cost and low-effort large area ordered 2D systems. An investigation of such PnC made out of a Si membrane loaded with a monolayer of silica microspheres was performed by means of TG technique in sub-GHz regime. ${ }^{[42]}$ The measured dispersion curves showed hybridization of Lamb waves with the microsphere-membrane contact resonance leading to low frequency band-gap. More recently, a micro-BLS study of similar PnCs of sub-micrometer polystyrene (PS) particles on $\mathrm{Si}_{3} \mathrm{~N}_{4}$ revealed a modification of GHz Lamb waves by the three discused schemes; period, local resonance and particle-membrane resonance. In addition to previous studies, micro-BLS revealed local resonance band gaps below and above the Bragg band gap. Thus, the operating frequency of such systems can be easily adjusted by geometrical means, i.e., particle size and material, lattice period and symmetry, membrane thickness and material or adhesion forces at particle-membrane and particle-particle interfaces (Figure $2(\mathrm{~d})) .{ }^{[79]}$

\section{Thermal Transport}

In this section, we focus on the use of PnCs for thermal transport management. We first describe the mechanisms affecting thermal transport, in particular in nanostructures, followed by experimental measurements in thin films and $\mathrm{PnCs}$ and finally we explain the thermal transport engineering possibilities of PnCs.

\subsection{Heat transport regimes}

When investigating thermal transport, the contribution of all phonons has to be taken into account. At thermal equilibrium, the phonon population follows the Bose-Einstein distribution, from which we can define a cut-off energy $k_{B} T$, where $k_{B}$ is the Boltzmann constant and $T$ the 
temperature. At $300 \mathrm{~K}$, this means that modes up to $\sim 6 \mathrm{THz}$ are populated and, therefore, can contribute to thermal transport. Hence, temperature is a crucial factor in the range of the phonon spectrum that needs to be addressed to alter thermal transport properties. To obtain the phonon contribution, other factors need to be taken into account, such as the phonon energy $\hbar \omega$ (where $\hbar$ is the reduced Planck's constant and $\omega$ is the angular frequency of each phonon mode), which decreases the contribution of very low frequency phonons.

Phonons are subjected to different scattering mechanisms such as phonon-phonon scattering processes $\left(\Gamma_{\mathrm{p}-\mathrm{p}}\right)$, impurity scattering $\left(\Gamma_{\mathrm{i}}\right)$ and boundary scattering $\left(\Gamma_{\mathrm{b}}\right)$. To estimate the phonon thermal conductivity in semiconductor material, knowledge of three major frequencydependent parameters is needed, that is, specific heat $(C)$, which also directly depends on temperature, phonon group velocity ( $v$ ), and the phonon mean free path (MFP) $\Lambda=v / \Gamma$, where $\Gamma$ is the effective or total phonon scattering rate. In general, $\Gamma$ is estimated as the sum of all the scattering rates corresponding to each scattering mechanism mentioned above assuming they are independent. ${ }^{[80]}$ The inverse of $\Gamma$ is the phonon relaxation time, i.e., the time that a phonon travels between scattering events. In the simple kinetic theory, the phonon thermal conductivity can then be calculated as:

$$
\kappa=\frac{1}{3} \sum_{\alpha} C_{\alpha} v_{\alpha .} \Lambda_{\alpha} C_{\alpha}
$$

where the summation is over all the phonons. ${ }^{[81]}$

Since phonon-phonon and impurity scattering are linked to the square and fourth power of the phonon frequencies, respectively, they become more relevant as the phonon frequency increases, i.e., as the temperature increases. While temperature does not affect the number of boundary scattering events, their nature changes drastically between pure diffuse scattering events at high temperatures, i.e., when the boundary roughness is comparable or larger than the phonon wavelength, and specular scattering, for which the momentum is conserved. At low temperature, the phonon population is shifted to longer wavelengths (lower frequencies) 
and the MFP of phonons increases, as well as the probability of specular scattering. If specular scattering is predominant over the diffusive one, thermal transport deviates from the classical Fourier law and non-diffusive (ballistic or quasi-ballistic) transport appears. ${ }^{[82,83]}$ In order that the wave-like behaviour of phonons emerges, for instance interference effects in PnCs ${ }^{[18,84,85]}$, the characteristic features of PnCs like the period should be smaller than the MFP. Therefore, the transport should not be limited by diffuse scattering at the interfaces between materials forming the PnC. In addition, the phase of the atomic displacements defining phonons needs to be maintained in a suitable spatial extension. This leads introducing the frequency-dependent phonon coherence length, which represents the average spatial extension of a phonon wave packet at the given frequency ${ }^{[86,87]}$. When phonon coherence length exceeds a required number of $\mathrm{PnC}$ periods, phonon wave interference modifies the energy dispersion relations of the phonon band structure. These deviations from diffusive transport are of critical importance in furthering our control over heat transport in nanostructures and can be said to constitute the objective of PnCs for thermal transport. To achieve this objective, the two main parameters that can be altered are the temperature, and the dimensions of the PnC. In the following, we will examine first classical size effects linked to diffusive thermal transport and then the impact of the phonon dispersion on heat transport through phonon interference effects.

\subsection{Diffusive phonon transport}

At room temperature, the dominant phonon wavelength in silicon is only a few nanometers and the MFPs in bulk reaches several hundreds of nanometer and up to several micrometer. ${ }^{[88]}$ In comparison, surface roughness is usually on the order of the phonon wavelengths and then most of the boundary and surface scattering events are diffusive. Indeed, the specularity parameter $\mathrm{p}$ can be described by Soffer's formula ${ }^{[89]}$ as:

$$
p=e^{\frac{-16 \pi^{2} \eta^{2}}{\lambda^{2}} \cos ^{2}(\psi)}
$$


where $\eta$ is the characteristic roughness, $\lambda$ the phonon wavelength and $\psi$ the incident angle. For $p=0$ scattering will be purely diffuse meaning that the phase information of phonons and their direction of propagation are lost during boundary scattering events. As a consequence, thermal conductivity in nanostructures and PnCs is mainly governed by diffuse scattering. In thin films, a direct link between their thickness and thermal conductivity has been observed, ${ }^{[33,90,91]}$ and is well-described by the Fuchs-Sondheimer model. ${ }^{[28,33]}$ An experimental reconstruction of the MFP contribution to thermal conductivity shows that the main contribution stems from phonons with MFPs in the $100 \mathrm{~nm}-10 \mu \mathrm{m}$ range ${ }^{[90]}$ in agreement with first-principles calculations. ${ }^{[92]}$ Beyond the ideal case, heat transport in ultra-thin silicon membranes (sub-20-nm) depends strongly upon the properties of the surface at the nanoscale, which is determined by processing and fabrication. The interplay of several factors influencing thermal transport in the membranes is demonstrated by means of controlled experiments and modelling: thickness, the presence of surface native oxide layers, and surface roughness. The influence of these factors is summarized in Figure 3 (a) and (b) and have been reported in our previous work. ${ }^{[34]}$ It is seen that the thermal conductivity decreases mainly as a consequence of enhanced surface effects related to surface native oxide layer and roughness. Molecular dynamics simulations showed that diffuse scattering at the rough surfaces masks coherent effects in the thermal transport.

However, the purpose of PnCs for thermal transport applications is to go beyond simple classical size effects and engineer thermal properties via the modification of the dispersion relation. As was described in the previous sections, the most common approach to realize 2D PnCs is to pattern circular holes in a suspended silicon film. It has been widely shown that the thermal conductivity of such structures drops compared to non-patterned membranes. In most cases, this further reduction in thermal conductivity is due to an increase in surface scattering that can be summarized by two parameters, namely the surface-tovolume ratio and the spacing between holes (neck size), i.e., the smallest width of the 
channels allowing phonon transport. ${ }^{[17,32,82,93-96]}$ Similarly to thin films, the roughness of the surfaces impacts surface scattering, and therefore thermal conductivity. ${ }^{[97]}$ Further experiments confirmed the importance of geometric parameters such as the filling fraction, surface-to-volume ratio or the neck size, but also demonstrated that a further tuning of the thermal conductivity is possible with PnCs with random hole positions, by varying the hole overlap, i.e., the number and relative position of the phonon channels. ${ }^{[37]}$

Impurity and phonon-phonon scattering processes are predominant in bulk material at room temperature, whereas the importance of boundary scattering increases as temperature decreases. At room temperature, patterning PnCs will therefore increase the relative importance of boundary scattering due to the increase in surface density. However, as phonon wavelengths are not much longer than the surface roughness, most boundary scattering events are diffusive. This ensures that the periodicity itself does not impact thermal transport via phonon interference effects, together with the fact that the characteristic sizes are comparable to phonon MFPs in the PnCs. This has been experimentally demonstrated by introducing disorder in the position of the holes. ${ }^{[19,36]}$ Coherent phonon transport would require surface roughness well below the phonon wavelength, otherwise the phase information is destroyed. These conditions are also satisfied as the temperature decreases since the probability of phonon-phonon scattering events decreases due to lower phonon frequencies, whereas the boundary scattering becomes more common for the same reason, i.e., longer phonon wavelengths and bulk MFP. Hence, the impact of nanopatterning is enhanced as the temperature decreases as can be seen from Figure 3 (c) in the case of various PnCs with different surface density. The rate of the decrease of the thermal conductivity with increasing boundary scattering, i.e. PnC surface density, is larger at $300 \mathrm{~K}$ than at $900 \mathrm{~K}$ as at high temperature phonon-phonon scattering dominates over boundary scattering. The decrease of the as measured and extracted intrinsic thermal conductivities as a function of the surface to volume ratio is shown in Figure 3 (d) at the temperature of $600 \mathrm{~K}$. 


\subsection{Non-diffusive phonon transport and breakdown of Fourier law}

As detailed in previous sections, current state of the art PnCs have dimensions down to several tens of nanometers with block-copolymer technologies ${ }^{[98,99]}$ and hundreds of nanometers via a top-down approach. ${ }^{[17,35]}$ At these dimensions and at room temperature, the surface roughness together with the PnC lattice's disorder suppresses wave-like effects and, therefore, diffusive thermal transport prevails. Since the further reduction of PnCs features size, with surface roughness below the phonon wavelengths, is presently challenging, the main strategy used so far with PnCs is to study thermal transport at low temperatures. Indeed, the dominant wavelength becomes more than $20 \mathrm{~nm}$ at $4 \mathrm{~K}$ and is even longer at sub-Kelvin temperatures. Geometry is a factor that has been used to prove non-diffusive thermal transport in suspended silicon structures ${ }^{[100-102]}$ demonstrating the key role of specular surface scattering at sub-Kelvin temperatures in thermal transport. In a direct analogy with PnCs aimed at hypersonic waves introduced in the previous sections, Maasilta et al. ${ }^{[18]}$ have shown the direct correlation between the thermal conductance, calculated from the modified phonon dispersion relation, and experimental measurements, as depicted in Figure 4. Compared to the unpatterned membrane, PnCs showed a much lower thermal conductance. As the thermally transported power is proportional to the group velocity multiplied by the density of states and the energy distribution, i.e., the energy of a phonon mode multiplied by its population, this work emphasizes the influence of the density of states (Figure 4(a)) and group velocity (Figure 4(b)) on the thermal conductance. They demonstrated that although the reduction of the thermal conductance is related to the modification of the band structure, it is not due to the existence of a band gap, but from the combination of changes of the group velocities and the density of states. They also observed that the $\mathrm{PnC}$ with larger period displayed lower thermal conductance than its smaller counterpart (Fig. 4(d)), confirming not only that the presence of a band gap does not necessarily lead to lower thermal conductance, but also that their finding 
could not be attributed to diffusive transport. In fact, in a recent work, ${ }^{[103]}$ the size range of periods has been increased, which allowed to detect a reversal of the monotonically decreasing trend in the conductance as a function of the period that might well be a crossover between coherent and incoherent regimes. This demonstration opened the path to an enhanced control of heat transport as will be discussed in Section 5. Decreasing further the phonon population in the $\mathrm{mK}$ range attempts were made to observe the quantization of heat ${ }^{[104,105]}$ for which both low temperatures and small dimensions are required. Despite a clear ballistic signature in these experiments, the debate is still ongoing over the observation of the quantum of thermal conductance.

\section{Prospects}

2D PnCs in surface or in plate-like structures have been the basis of well-stablished control of the wave propagation and confinement based in the Bragg scattering and local resonance mechanisms. These developments have been usually originated from the analogy with concepts brought by advances in electronics and photonics. ${ }^{[53]}$ However, phonon propagation in 2D PnCs based waveguides and 2D PnCs based in non-metallic local resonators in Sicompatible electro-acoustic platforms operating at about $1 \mathrm{GHz}$ have been only demonstrated recently. [11,106] This work provides a prospect towards reduction of losses and key applications in the next generation of wireless communication devices. A different mechanism than the one presented in Section 3 (i.e. coherent phonon scattering in a periodic arrangement of scatterers) that could be applied in the control of elastic waves is the phenomenon of multiple-scattering in disordered structures, including Anderson-localization of the elastic field. It has been already measured in a 3D elastic disordered structure in the ultrasound, ${ }^{[107]}$ and in a vertical superlattice in the hypersound, ${ }^{[108]}$ but presently there has been no observation in $2 \mathrm{D}$ structures.

Topological elasticity is an emerging field of potentially transformative impact. ${ }^{[109]}$ Besides the fact that elastic waves provide a suitable platform to probe unique phenomena 
related to the concept of topology, the control of elastic waves propagation can benefit the functionalities like free-edge backscattering waveguiding. Breaking the time-reversal symmetry to obtain an equivalent to the quantum Hall effect in bosonic lossless systems requires active materials responding to an external field, a nonlinearity or time-dependent physical properties ${ }^{[110]}$, which in the case of elasticity is especially difficult. Therefore several proposals, as well as few experimental realizations, have achieved topological phenomena by emulating the quantum spin Hall effect ${ }^{[111-116]}$ or quantum valley Hall effect ${ }^{[117-120]}$ in passive linear structures. While conserving time-reversal symmetry, these topological structures are based on breaking some spatial inversion symmetry. The interface between two lattices showing topological phase transition produced by band inversion supports topological edge states. Although the proposed designs seem to be unaffected by sharp geometric features to a large extent, the waveguides formed from topological edge states are not unidirectional in the strict sense (mirror symmetry breaking only). Therefore, there is the need to study at which level the edge states are immune to disorder introduced, for example, during the fabrication process. The achievement of a specific wave dispersion relation and the necessary conditions to obtain the topological states in the $\mathrm{GHz}$ range is challenging due to the inherent complexity of elastic waves.

Currently, SAW as well as membrane-based PnCs suffers from the absence of coherent sources of phonons breaking the threshold of few GHz. The game changer which will allow using the wealth of PnCs features is expected from the structures being both phononic and photonic, i.e., phoXonic or optomechanical (OM) crystals. ${ }^{[121]}$ The different electromagnetic and elastic wave velocities allow the localization of both types of waves in the same length scale, despite their very different frequencies. Therefore, the characteristic sizes of PnCs that modify the phonon dispersion relations in the $\mathrm{GHz}$ range modify simultaneously the photon dispersion relation for similar wavelengths but at much higher frequencies. An OM cavity can be created by introducing a defect in the OM crystals, where 
the co-localization of light and vibrational motion in a small volume can greatly enhance the optomechanical coupling. ${ }^{[122-124]}$ Elastic modes provide an adaptable approach to support coherent coupling between different particles and quasiparticles, which would form the basis of an extended range of procedures to generate, drive and detect waves. This will enable new signal transduction architectures and signal-processing functionalities like optical field manipulation of RF waves by means of elastic interfaces. ${ }^{[125]}$ Moreover, elastic-wave signalprocessing in hybrid electro-opto-mechanical platforms, where new functionalities of PnCs can be derived based on non-linear effects, complex dynamics and topological effects, opens the door to the new field of applied phononics.

PnCs offer numerous prospects in thermal management. The challenge lies in the broad spectrum of high frequency phonons that carry heat, making it harder to control. Nonetheless, various applications have been suggested, among which thermal circuits and energy applications have promising prospects. A recent study has shown the importance of specular surface scattering of phonons for thermal circuits, with the experimental demonstration of a thermal lens ${ }^{[16]}$. This work demonstrates the possibility to guide and focus heat onto predefined areas as a premise to thermal circuitry. The development of different phononic components, as for example thermal diodes, is currently a growing research area of great interest. ${ }^{[126,127]}$ In addition, whereas some applications have already been demonstrated experimentally such as thermal cloaking ${ }^{[128,129]}$, achieving a similar effect with PnCs is a challenge.

To further increase the efficiency of PnCs, operating at lower temperature is not a general solution. We have shown that decreasing the feature size of PnCs would increase the operating frequency, but simple patterning is limited due to the minimum surface roughness achievable. To remedy these issues, a multiscale approach has been proposed to filter phonons and suppress the contribution of high-frequency phonons with impurities, alloying or grain boundaries $^{[130]}$. In practice, this approach modifies the range of frequencies of phonons 
carrying heat towards a lower and narrower range that is, therefore, easier to manipulate. This approach is particularly interesting to reduce thermal conductivity and thus to recuperate waste heat with thermoelectric energy harvesters ${ }^{[131]}$, for instance. Overall, we have shown that PnCs have a strong potential for thermal applications despite the difficulties arising from their broad spectrum involved that requires further technological and material developments to achieve practical figures of merit.

Acknowledgements

ICN2 is supported by Spanish MINECO (Severo Ochoa Centers of Excellence Program under Grant SEV-2017-0706), and by the Generalitat de Catalunya (Grant 2017SGR806 and the CERCA Program). B.G. acknowledges the support from the Foundation for Polish Science (POIR.04.04.00-00-5D1B/18), Polish National Science Centre (UMO-2018/31/D/ST3/03882) and ERC AdG SmartPhon (Grant No. 694977).

\section{References}

[1] P. YU, M. Cardona, Fundamentals of Semiconductors: Physics and Materials Properties; Graduate Texts in Physics; 4th ed.; Springer-Verlag: Berlin Heidelberg, 2010.

[2] J. M. Ziman, Principles of the Theory of Solids by J. M. Ziman. Cambridge Core 1972.

[3] S. Volz, J. Ordonez-Miranda, A. Shchepetov, M. Prunnila, J. Ahopelto, T. Pezeril, G. Vaudel, V. Gusev, P. Ruello, E. M. Weig, M. Schubert, M. Hettich, M. Grossman, T. Dekorsy, F. Alzina, B. Graczykowski, E. Chavez-Angel, J. Sebastian Reparaz, M. R. Wagner, C. M. Sotomayor-Torres, S. Xiong, S. Neogi, D. Donadio, Eur. Phys. J. B 2016, 89, 15.

[4] J. L. Rose, Ultrasonic Guided Waves in Solid Media by Joseph L. Rose. Cambridge Core 2014.

[5] C. M. S. Torres, A. Zwick, F. Poinsotte, J. Groenen, M. Prunnila, J. Ahopelto, A. Mlayah, V. Paillard, physica status solidi (c) 2004, 1, 2609.

[6] R. S. Bandhu, X. Zhang, R. Sooryakumar, K. Bussmann, Phys. Rev. B 2004, 70, 075409.

[7] J. Cuffe, E. Chávez, A. Shchepetov, P.-O. Chapuis, E. H. El Boudouti, F. Alzina, T. Kehoe, J. Gomis-Bresco, D. Dudek, Y. Pennec, B. Djafari-Rouhani, M. Prunnila, J. Ahopelto, C. M. Sotomayor Torres, Nano Lett. 2012, 12, 3569.

[8] C. Sumanya, J. D. Comins, A. G. Every, Wave Motion 2017, 68, 78.

[9] M. Sigalas, E. N. Economou, Solid State Communications 1993, 86, 141.

[10] M. S. Kushwaha, P. Halevi, L. Dobrzynski, B. Djafari-Rouhani, Phys. Rev. Lett. 1993, 71, 2022.

[11] R. Pourabolghasem, R. Dehghannasiri, A. A. Eftekhar, A. Adibi, Phys. Rev. Applied 2018, 9 , 014013.

[12] V. L. Zhang, C. G. Hou, H. H. Pan, F. S. Ma, M. H. Kuok, H. S. Lim, S. C. Ng, M. G. Cottam, M. Jamali, H. Yang, Appl. Phys. Lett. 2012, 101, 053102. 
[13] C. G. Hou, V. L. Zhang, S. C. Ng, M. H. Kuok, H. S. Lim, X. M. Liu, A. O. Adeyeye, Appl. Phys. Lett. 2014, 104, 093108.

[14] B. Graczykowski, M. Sledzinska, F. Alzina, J. Gomis-Bresco, J. S. Reparaz, M. R. Wagner, C. M. Sotomayor Torres, Phys. Rev. B 2015, 91, 075414.

[15] Cavity Optomechanics: Nano- and Micromechanical Resonators Interacting with Light; Aspelmeyer, M.; Kippenberg, T. J.; Marquardt, F., Eds.; Quantum Science and Technology; Springer-Verlag: Berlin Heidelberg, 2014.

[16] R. Anufriev, A. Ramiere, J. Maire, M. Nomura, Nature Communications 2017, 8, 15505.

[17] B. Graczykowski, A. E. Sachat, J. S. Reparaz, M. Sledzinska, M. R. Wagner, E. ChavezAngel, Y. Wu, S. Volz, Y. Wu, F. Alzina, C. M. S. Torres, Nature Communications 2017, 8, 415.

[18] N. Zen, T. A. Puurtinen, T. J. Isotalo, S. Chaudhuri, I. J. Maasilta, Nature Communications 2014, 5, 3435.

[19] J. Maire, R. Anufriev, R. Yanagisawa, A. Ramiere, S. Volz, M. Nomura, Science Advances 2017, 3, e1700027.

[20] A. Mavrokefalos, M. T. Pettes, F. Zhou, L. Shi, Review of Scientific Instruments 2007, 78, 034901.

[21] A. V. Feshchenko, L. Casparis, I. M. Khaymovich, D. Maradan, O.-P. Saira, M. Palma, M. Meschke, J. P. Pekola, D. M. Zumbühl, Phys. Rev. Applied 2015, 4, 034001.

[22] D. G. Cahill, P. V. Braun, G. Chen, D. R. Clarke, S. Fan, K. E. Goodson, P. Keblinski, W. P. King, G. D. Mahan, A. Majumdar, H. J. Maris, S. R. Phillpot, E. Pop, L. Shi, Applied Physics Reviews 2014, 1, 011305.

[23] J. S. Reparaz, E. Chavez-Angel, M. R. Wagner, B. Graczykowski, J. Gomis-Bresco, F. Alzina, C. M. Sotomayor Torres, Review of Scientific Instruments 2014, 85, 034901.

[24] B. Graczykowski, M. Sledzinska, N. Kehagias, F. Alzina, J. S. Reparaz, C. M. Sotomayor Torres, Appl. Phys. Lett. 2014, 104, 123108.

[25] S. Mielcarek, A. Trzaskowska, B. Graczykowski, J. Sarkar, physica status solidi (RRL) Rapid Research Letters 2012, 6, 175.

[26] D. Nardi, M. Travagliati, M. E. Siemens, Q. Li, M. M. Murnane, H. C. Kapteyn, G. Ferrini, F. Parmigiani, F. Banfi, Nano Lett. 2011, 11, 4126.

[27] B. Graczykowski, S. Mielcarek, A. Trzaskowska, J. Sarkar, P. Hakonen, B. Mroz, Phys. Rev. B 2012, 86, 085426.

[28] A. M. Marconnet, M. Asheghi, K. E. Goodson, Journal of Heat Transfer 2013, 135, 061601.

[29] A. Shchepetov, M. Prunnila, F. Alzina, L. Schneider, J. Cuffe, H. Jiang, E. I. Kauppinen, C. M. Sotomayor Torres, J. Ahopelto, Appl. Phys. Lett. 2013, 102, 192108.

[30] S. Mohammadi, A. A. Eftekhar, A. Khelif, W. D. Hunt, A. Adibi, Appl. Phys. Lett. 2008, 92, 221905.

[31] S. Mohammadi, A. A. Eftekhar, W. D. Hunt, A. Adibi, Appl. Phys. Lett. 2009, 94, 051906.

[32] R. Anufriev, M. Nomura, Physical Review B 2016, 93, 045410.

[33] E. Chavez-Angel, J. S. Reparaz, J. Gomis-bresco, M. R. Wagner, J. Cuffe, B. Graczykowski, A. Shchepetov, H. Jiang, M. Prunnila, J. Ahopelto, F. Alzina, C. M. Sotomayor Torres, APL Materials 2014, 2, 012113.

[34] S. Neogi, J. S. Reparaz, L. F. C. Pereira, B. Graczykowski, M. R. Wagner, M. Sledzinska, A. Shchepetov, M. Prunnila, J. Ahopelto, C. M. Sotomayor-Torres, D. Donadio, ACS Nano 2015, 9, 3820.

[35] M. Sledzinska, B. Graczykowski, F. Alzina, J. Santiso Lopez, C. M. Sotomayor Torres, Microelectronic Engineering 2016, 149, 41.

[36] M. R. Wagner, B. Graczykowski, J. S. Reparaz, A. El Sachat, M. Sledzinska, F. Alzina, C. M. Sotomayor Torres, Nano Lett. 2016, 16, 5661.

[37] M. Sledzinska, B. Graczykowski, F. Alzina, U. Melia, K. Termentzidis, D. Lacroix, C. M. S. Torres, Nanotechnology 2019. 
[38] D. F. Goettler, M. F. Su, C. M. Reinke, S. Alaie, P. E. Hopkins, R. H. Olsson, I. El-Kady, Z. C. Leseman, AIP Advances 2011, 1, 042001.

[39] Y. Achaoui, A. Khelif, S. Benchabane, L. Robert, V. Laude, Phys. Rev. B 2011, 83, 104201.

[40] A. Boes, V. Sivan, G. Ren, D. Yudistira, S. Mailis, E. Soergel, A. Mitchell, Appl. Phys. Lett. 2015, 107, 022901.

[41] D. Yudistira, A. Boes, B. Graczykowski, F. Alzina, L. Y. Yeo, C. M. Sotomayor Torres, A. Mitchell, Phys. Rev. B 2016, 94, 094304.

[42] A. Khanolkar, S. Wallen, M. Abi Ghanem, J. Jenks, N. Vogel, N. Boechler, Appl. Phys. Lett. 2015, 107, 071903.

[43] K. B. Blodgett, J. Am. Chem. Soc. 1934, 56, 495.

[44] N. Vogel, M. Retsch, C.-A. Fustin, A. del Campo, U. Jonas, Chem. Rev. 2015, 115, 6265.

[45] S. Park, D. H. Lee, J. Xu, B. Kim, S. W. Hong, U. Jeong, T. Xu, T. P. Russell, Science 2009, 323, 1030.

[46] J. Lim, H.-T. Wang, J. Tang, S. C. Andrews, H. So, J. Lee, D. H. Lee, T. P. Russell, P. Yang, ACS Nano 2016, 10, 124.

[47] C. Simão, W. Khunsin, N. Kehagias, M. Salaun, M. Zelsmann, M. A. Morris, C. M. S. Torres, Nanotechnology 2014, 25, 175703.

[48] M. Verdier, D. Lacroix, S. Didenko, J.-F. Robillard, E. Lampin, T.-M. Bah, K. Termentzidis, Phys. Rev. B 2018, 97, 115435.

[49] W. Bogaerts, R. Baets, P. Dumon, V. Wiaux, S. Beckx, D. Taillaert, B. Luyssaert, J. V. Campenhout, P. Bienstman, D. V. Thourhout, Journal of Lightwave Technology 2005, 23, 401.

[50] T. Asano, Y. Ochi, Y. Takahashi, K. Kishimoto, S. Noda, Opt. Express, OE 2017, 25, 1769.

[51] M. Borselli, T. J. Johnson, O. Painter, Appl. Phys. Lett. 2006, 88, 131114.

[52] J. Cuffe, D. Dudek, N. Kehagias, P.-O. Chapuis, V. Reboud, F. Alsina, J. G. McInerney, C. M. Sotomayor Torres, Microelectronic Engineering 2011, 88, 2233.

[53] Y. Pennec, J. O. Vasseur, B. Djafari-Rouhani, L. Dobrzyński, P. A. Deymier, Surface Science Reports 2010, 65, 229.

[54] A. Khelif, A. Adibi, Phononic Crystals: Fundamentals and Applications; Springer, 2015.

[55] V. Laude, Phononic Crystals: Artificial Crystals for Sonic, Acoustic, and Elastic Waves; Walter de Gruyter GmbH \& Co KG, 2015.

[56] P. A. Deymier, Acoustic Metamaterials and Phononic Crystals; Springer Science \& Business Media, 2013.

[57] C. Campbell, Surface Acoustic Wave Devices for Mobile and Wireless Communications, Four-Volume Set; Academic Press, 1998.

[58] W.- Bulst, G. Fischerauer, L. Reindl, IEEE Transactions on Industrial Electronics 2001, 48, 265.

[59] M. Maldovan, Nature Materials 2015, 14, 667.

[60] A. H. Safavi-Naeini, J. T. Hill, S. Meenehan, J. Chan, S. Gröblacher, O. Painter, Phys. Rev. Lett. 2014, 112, 153603.

[61] E. Gavartin, R. Braive, I. Sagnes, O. Arcizet, A. Beveratos, T. J. Kippenberg, I. Robert-Philip, Phys. Rev. Lett. 2011, 106, 203902.

[62] Y. Tanaka, S. Tamura, Phys. Rev. B 1998, 58, 7958.

[63] J.-F. Robillard, A. Devos, I. Roch-Jeune, Phys. Rev. B 2007, 76, 092301.

[64] C. Giannetti, B. Revaz, F. Banfi, M. Montagnese, G. Ferrini, F. Cilento, S. Maccalli, P. Vavassori, G. Oliviero, E. Bontempi, L. E. Depero, V. Metlushko, F. Parmigiani, Phys. Rev. B 2007, 76, 125413.

[65] A. Trzaskowska, S. Mielcarek, B. Graczykowski, B. Mroz, P. Patoka, M. Giersig, Journal of Alloys and Compounds 2012, 527, 96.

[66] A. Khelif, B. Aoubiza, S. Mohammadi, A. Adibi, V. Laude, Phys. Rev. E 2006, 74, 046610. 
[67] Y. Pennec, B. Djafari-Rouhani, H. Larabi, J. O. Vasseur, A. C. Hladky-Hennion, Phys. Rev. B 2008, 78, 104105.

[68] Wright Oliver B., Matsuda Osamu, Philosophical Transactions of the Royal Society A: Mathematical, Physical and Engineering Sciences 2015, 373, 20140364.

[69] T.-W. Liu, Y.-C. Tsai, Y.-C. Lin, T. Ono, S. Tanaka, T.-T. Wu, AIP Advances 2014, 4, 124201.

[70] R. Dehghannasiri, A. A. Eftekhar, A. Adibi, Phys. Rev. Applied 2018, 10, 064019.

[71] N. Boechler, J. K. Eliason, A. Kumar, A. A. Maznev, K. A. Nelson, N. Fang, Phys. Rev. Lett. 2013, 111, 036103.

[72] M. Hiraiwa, M. Abi Ghanem, S. P. Wallen, A. Khanolkar, A. A. Maznev, N. Boechler, Phys. Rev. Lett. 2016, 116, 198001.

[73] P. H. Otsuka, S. Mezil, O. Matsuda, M. Tomoda, A. A. Maznev, T. Gan, N. Fang, N. Boechler, V. E. Gusev, O. B. Wright, New J. Phys. 2018, 20, 013026.

[74] A. Vega-Flick, R. A. Duncan, S. P. Wallen, N. Boechler, C. Stelling, M. Retsch, J. J. Alvarado-Gil, K. A. Nelson, A. A. Maznev, Phys. Rev. B 2017, 96, 024303.

[75] T. Makkonen, V. P. Plessky, W. Steichen, M. M. Salomaa, Appl. Phys. Lett. 2003, 82, 3351.

[76] B. Graczykowski, F. Alzina, J. Gomis-Bresco, C. M. Sotomayor Torres, Journal of Applied Physics 2016, 119, 025308.

[77] R. Pourabolghasem, A. Khelif, S. Mohammadi, A. A. Eftekhar, A. Adibi, Journal of Applied Physics 2014, 116, 013514.

[78] M. Nomura, J. Nakagawa, Y. Kage, J. Maire, D. Moser, O. Paul, Appl. Phys. Lett. 2015, 106, 143102.

[79] B. Graczykowski et al., Unpublished.

[80] M. G. Holland, Phys. Rev. 1963, 132, 2461.

[81] Thermal Conductivity - Theory, Properties, and Applications | Terry M. Tritt | Springer.

[82] J. Lee, J. Lim, P. Yang, Nano Letters 2015, 15, 3273.

[83] J. A. Johnson, A. A. Maznev, J. Cuffe, J. K. Eliason, A. J. Minnich, T. Kehoe, C. M. S. Torres, G. Chen, K. A. Nelson, Phys. Rev. Lett. 2013, 110, 025901.

[84] N. K. Ravichandran, A. J. Minnich, Physical Review B 2014, 89, 205432.

[85] J. Ravichandran, A. K. Yadav, R. Cheaito, P. B. Rossen, A. Soukiassian, S. J. Suresha, J. C. Duda, B. M. Foley, C.-H. Lee, Y. Zhu, A. W. Lichtenberger, J. E. Moore, D. a Muller, D. G. Schlom, P. E. Hopkins, A. Majumdar, R. Ramesh, M. a Zurbuchen, Nature materials 2014, $13,168$.

[86] B. Latour, S. Volz, Y. Chalopin, Physical Review B - Condensed Matter and Materials Physics 2014, 90, 1.

[87] J. Ma, J. S. Sadhu, D. Ganta, H. Tian, S. Sinha, AIP Advances 2014, 4.

[88] K. T. Regner, D. P. Sellan, Z. Su, C. H. Amon, A. J. H. Mcgaughey, J. A. Malen, Nature Communications 2013, 4, 1640.

[89] S. B. Soffer, Journal of Applied Physics 1967, 38, 1710.

[90] J. Cuffe, J. K. Eliason, A. A. Maznev, K. C. Collins, J. a. Johnson, A. Shchepetov, M. Prunnila, J. Ahopelto, C. M. Sotomayor Torres, G. Chen, K. a. Nelson, Physical Review B 2015, 91, 245423.

[91] A. Vega-Flick, R. A. Duncan, J. K. Eliason, J. Cuffe, J. A. Johnson, J. P. M. Peraud, L. Zeng, Z. Lu, A. A. Maznev, E. N. Wang, J. J. Alvarado-Gil, M. Sledzinska, C. M. Sotomayor Torres, G. Chen, K. A. Nelson, AIP Advances 2016, 6.

[92] K. Esfarjani, G. Chen, H. T. Stokes, Phys. Rev. B 2011, 84, 085204.

[93] C. M. Reinke, M. F. Su, B. L. Davis, B. Kim, M. I. Hussein, Z. C. Leseman, R. H. Olsson-Iii, I. El-Kady, AIP Advances 2011.

[94] P. E. Hopkins, C. M. Reinke, M. F. Su, R. H. Olsson, E. A. Shaner, Z. C. Leseman, J. R. Serrano, L. M. Phinney, I. El-Kady, Nano Letters 2011, 11, 107. 
[95] J.-K. Yu, S. Mitrovic, D. Tham, J. Varghese, J. R. Heath, Nature nanotechnology 2010, 5, 718.

[96] S. Alaie, D. F. Goettler, M. Su, Z. C. Leseman, C. M. Reinke, I. El-Kady, Nature Communications 2015, 6, 7228.

[97] R. Yanagisawa, J. Maire, A. Ramiere, R. Anufriev, M. Nomura, Applied Physics Letters 2017, $110,133108$.

[98] J. Tang, H. T. Wang, D. H. Lee, M. Fardy, Z. Huo, T. P. Russell, P. Yang, Nano Letters 2010, $10,4279$.

[99] M. Salaun, M. Zelsmann, S. Archambault, D. Borah, N. Kehagias, C. Simao, O. Lorret, M. T. Shaw, C. M. Sotomayor Torres, M. A. Morris, Journal of Materials Chemistry C 2013, 1 , 3544.

[100] J. S. Heron, C. Bera, T. Fournier, N. Mingo, O. Bourgeois, Physical Review B - Condensed Matter and Materials Physics 2010, 82, 1.

[101] C. Blanc, A. Rajabpour, S. Volz, T. Fournier, O. Bourgeois, Applied Physics Letters 2013, 103.

[102] O. Bourgeois, D. Tainoff, A. Tavakoli, Y. Liu, C. Blanc, M. Boukhari, A. Barski, E. Hadji, Comptes Rendus Physique 2016, 17, 1154.

[103] Y. Tian, T. A. Puurtinen, Z. Geng, I. J. Maasilta, Phys. Rev. Applied 2019, 12, 014008.

[104] K. Schwab, E. A. E. Henriksen, J. M. Worlock, M. L. M. Roukes, Nature 2000, 404, 974.

[105] A. Tavakoli, K. Lulla, T. Crozes, N. Mingo, E. Collin, O. Bourgeois, Nature Communications 2018, 9, 4287.

[106] R. Dehghannasiri, A. A. Eftekhar, A. Adibi, Phys. Rev. Applied 2018, 10, 064019.

[107] H. Hu, A. Strybulevych, J. H. Page, S. E. Skipetrov, B. A. van Tiggelen, Nature Physics 2008, $4,945$.

[108] G. Arregui, N. D. Lanzillotti-Kimura, C. M. Sotomayor-Torres, P. D. García, Phys. Rev. Lett. 2019, 122, 043903.

[109] S. D. Huber, Nature Physics 2016, 12, 621.

[110] D. Jalas, A. Petrov, M. Eich, W. Freude, S. Fan, Z. Yu, R. Baets, M. Popović, A. Melloni, J.

D. Joannopoulos, M. Vanwolleghem, C. R. Doerr, H. Renner, Nature Photonics 2013, 7, 579.

[111] S. H. Mousavi, A. B. Khanikaev, Z. Wang, Nature Communications 2015, 6, 8682.

[112] S.-Y. Yu, C. He, Z. Wang, F.-K. Liu, X.-C. Sun, Z. Li, H.-Z. Lu, M.-H. Lu, X.-P. Liu, Y.-F. Chen, Nature Communications 2018, 9, 3072.

[113] R. Chaunsali, C.-W. Chen, J. Yang, Phys. Rev. B 2018, 97, 054307.

[114] H. Chen, H. Nassar, A. N. Norris, G. K. Hu, G. L. Huang, Phys. Rev. B 2018, 98, 094302.

[115] Z. Yu, Z. Ren, J. Lee, Scientific Reports 2019, 9, 1805.

[116] T. Lee, H. Iizuka, Phys. Rev. B 2019, 99, 064305.

[117] R. K. Pal, M. Ruzzene, New J. Phys. 2017, 19, 025001.

[118] J. Vila, R. K. Pal, M. Ruzzene, Phys. Rev. B 2017, 96, 134307.

[119] T.-W. Liu, F. Semperlotti, Phys. Rev. Applied 2018, 9, 014001.

[120] M. Yan, J. Lu, F. Li, W. Deng, X. Huang, J. Ma, Z. Liu, Nature Materials 2018, 17, 993.

[121] D. Navarro-Urrios, J. Gomis-Bresco, F. Alzina, N. E. Capuj, P. D. García, M. F. Colombano, E. Chavez-Angel, C. M. Sotomayor-Torres, J. Opt. 2016, 18, 094006.

[122] M. Eichenfield, J. Chan, R. M. Camacho, K. J. Vahala, O. Painter, Nature 2009, 462, 78.

[123] J. Chan, A. H. Safavi-Naeini, J. T. Hill, S. Meenehan, O. Painter, Appl. Phys. Lett. 2012, 101, 081115.

[124] J. Gomis-Bresco, D. Navarro-Urrios, M. Oudich, S. El-Jallal, A. Griol, D. Puerto, E. Chavez, Y. Pennec, B. Djafari-Rouhani, F. Alzina, A. Martínez, C. M. S. Torres, Nature Communications 2014, 5, 4452.

[125] K. C. Balram, M. I. Davanço, J. D. Song, K. Srinivasan, Nature Photonics 2016, 10, 346.

[126] S. R. Sklan, AIP Advances 2015, 5, 053302.

[127] R. Dettori, C. Melis, R. Rurali, L. Colombo, Journal of Applied Physics 2016, 119, 215102. 
[128] C. Z. Fan, Y. Gao, J. P. Huang, Applied Physics Letters 2008, 92, 251907.

[129] S. Narayana, Y. Sato, Physical Review Letters 2012, 108, 214303.

[130] M. Maldovan, Physical Review Letters 2013, 110, 025902.

[131] M. Maldovan, Nature 2013, 503, 209.
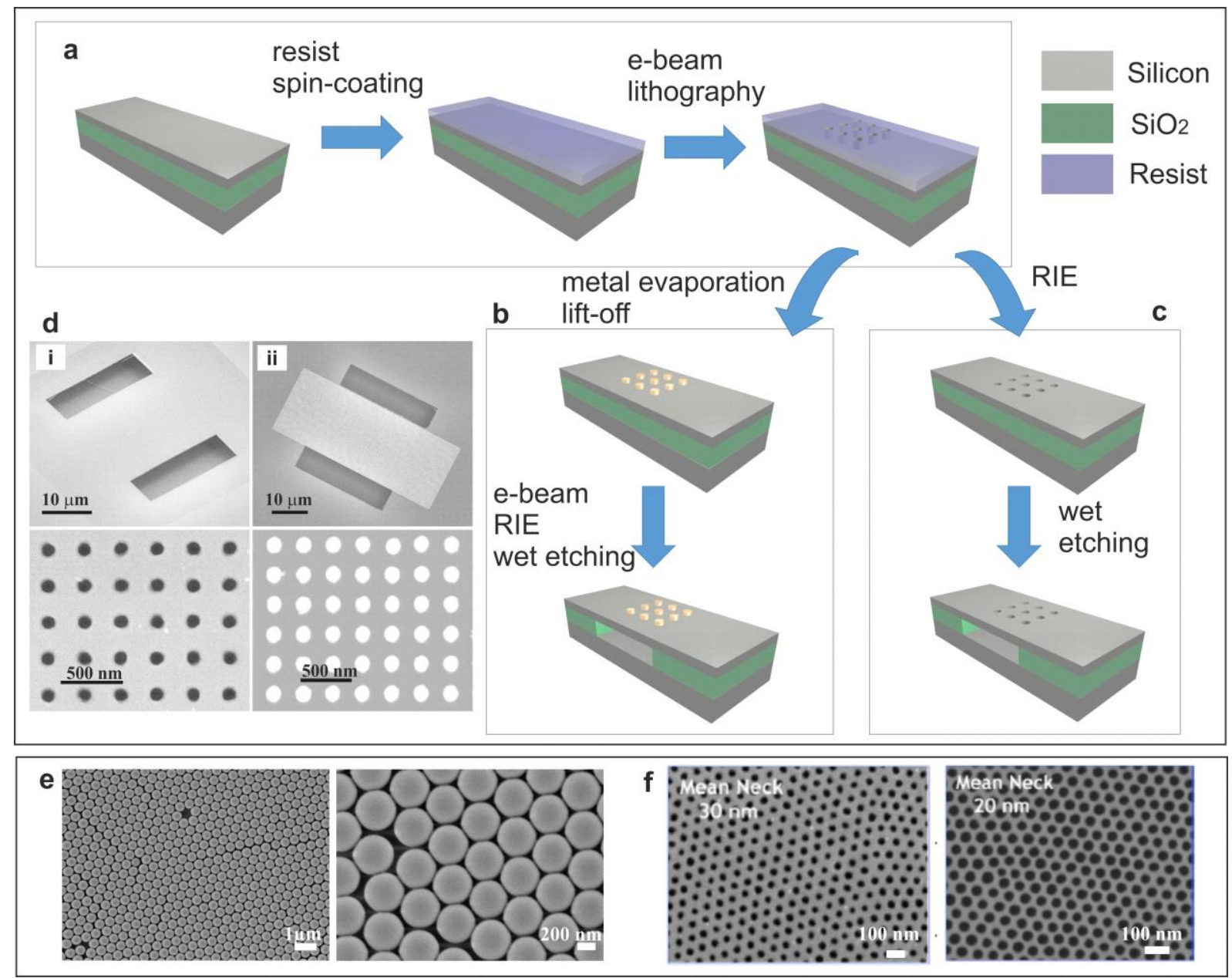

Figure 1 (a) Schematic of the fabrication process for free-standing PnCs in the SOI wafers (b) with metallic pillars (c) with holes (d) Examples of free-standing PnCs with (i) holes and (ii) pillars. Reproduced with permission. ${ }^{[14]}$ Copyright 2015, American Physical Society. (e) Representative image of the polystyrene microsphere monolayer on a $\mathrm{Si}_{3} \mathrm{~N}_{4}$ surface. ${ }^{[79]}$ (f) 
Holey silicon fabricated using BCP self-assembly and RIE Reproduced with permission. ${ }^{[46]}$ Copyright 2015, American Chemical Society.

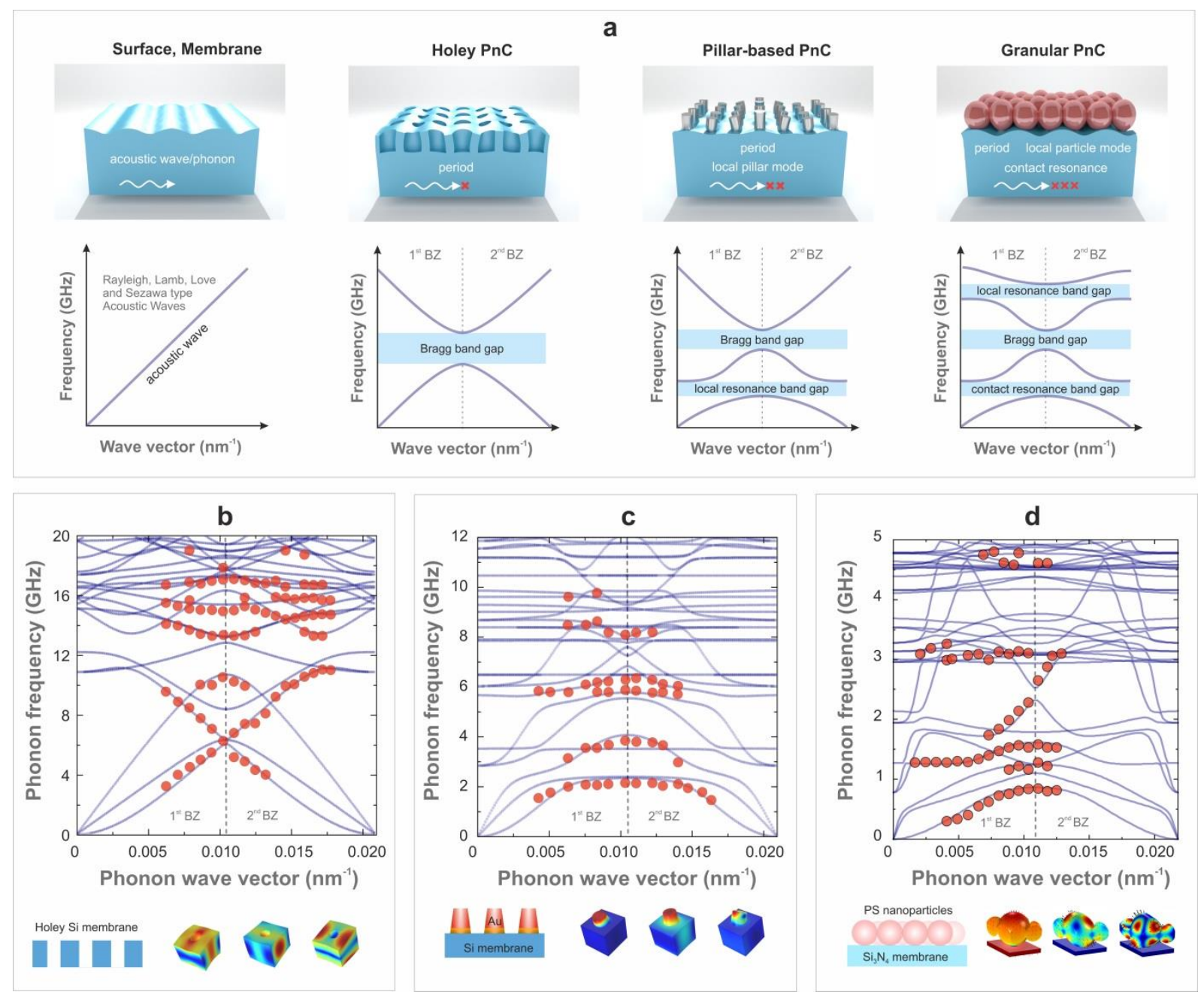

Figure 2 (a, upper panels) Schematic presentation of three types of 2D PnCs and (a, lower panels) corresponding phonon dispersion relations. Holey, pillar-based and granular 2D PnCs are compared with unperturbed surface/membrane. (b-d) Exemplary phonon dispersion relations measured (circles) and calculated (lines). Experimental data was obtained by means of BLS while theoretical fits were calculated using elastic continuum FEM model. Panels (b) and (c) depict dispersions for holey and pillar-based square lattice PnCs, respectively, made on thin Si membrane. Reproduced with permission. ${ }^{[14]}$ Copyright 2015, American Physical Society.Panel (c) shows dispersion of granular PnCs made out of a monolayer of close-packed PS nanoparticles deposited on ultra-thin $\mathrm{Si}_{3} \mathrm{~N}_{4}$ membrane. ${ }^{[79]}$ 

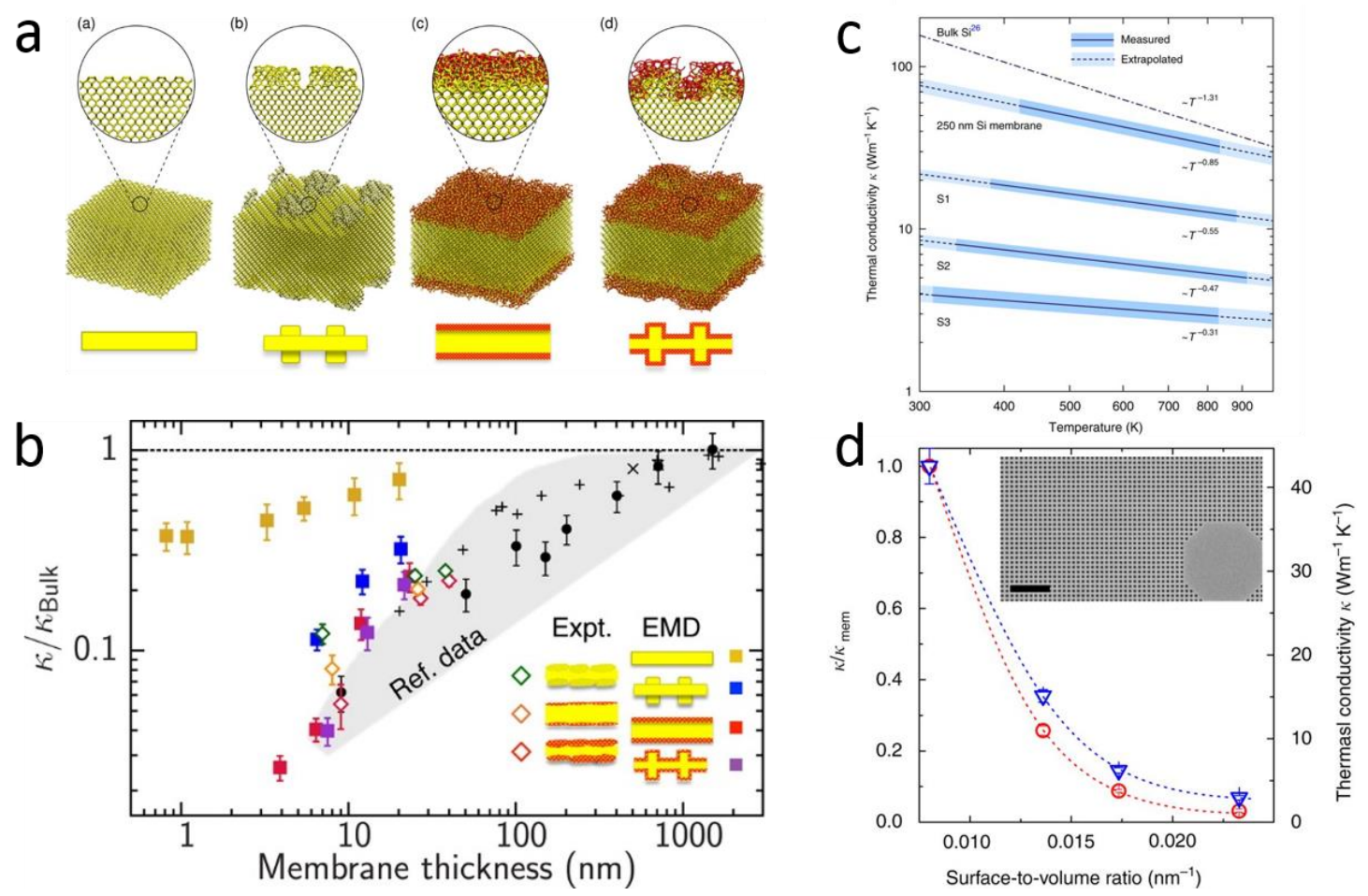

Figure 3. Thermal transport measurements in the diffusive regime. (a) Schematic representation of the different membranes under study. (b) Thermal conductivity measurements as a function of membrane thickness for different types of surfaces. Reproduced with permission. ${ }^{[34]}$ Copyright 2015, American Chemical Society. (c) Thermal conductivity of PnCs as a function of temperature and surface-to-volume ratio. (d) Measured (red circles) and intrinsic (blue triangles) thermal conductivities as a function of the surfaceto-volume ratio at a temperature of $600 \mathrm{~K}^{[17]}$ 

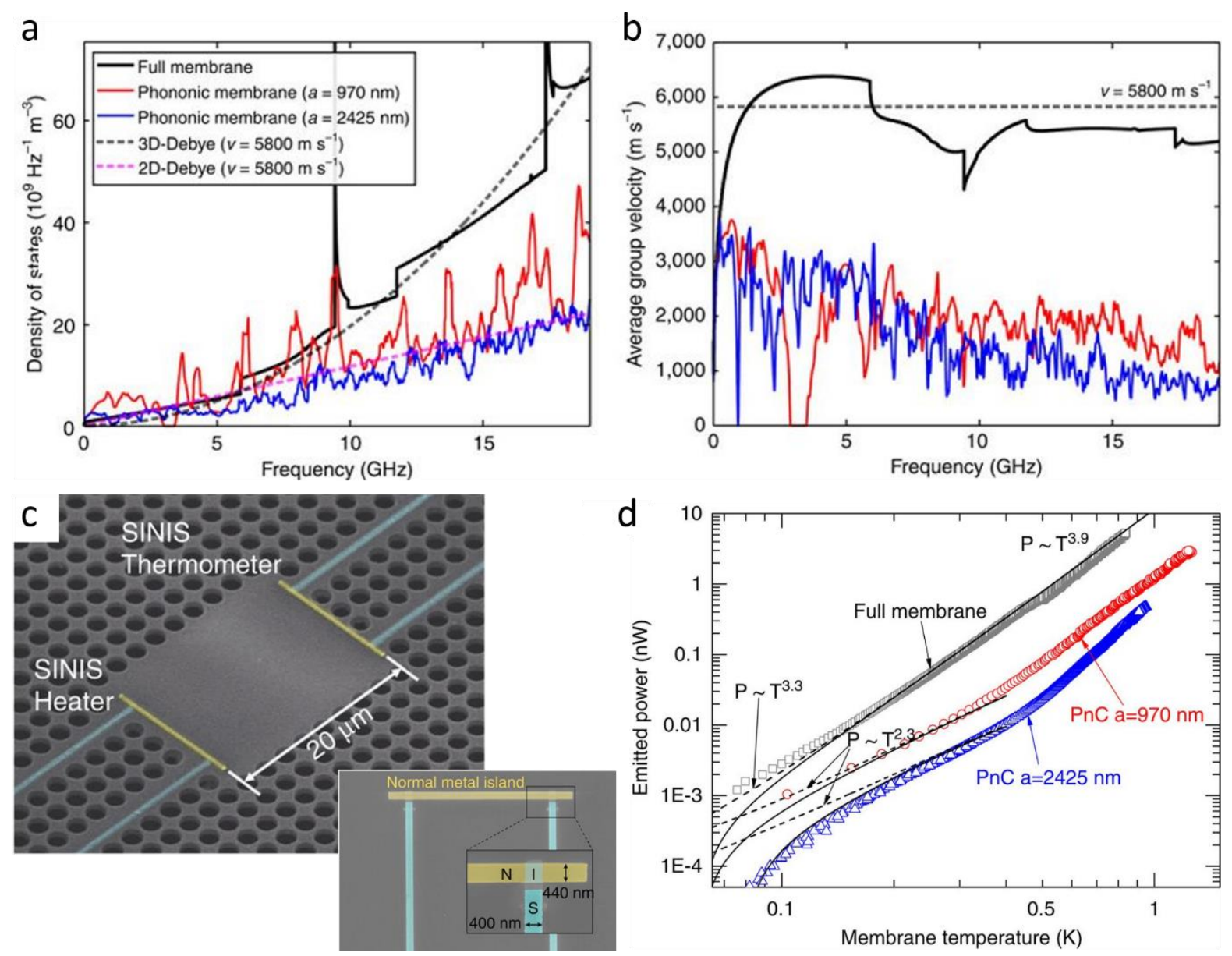

Figure 4. Non-diffusive thermal transport measurements. Phonon density of state (a) and group velocity (b) calculated from the phonon dispersion relation for a full unpatterned membrane and two phononic crystals of pitch $970 \mathrm{~nm}$ (red) and $2425 \mathrm{~nm}$ (blue). (c) SEM image of the calculated and measured phononic crystal and measurement system. (d) Ultralow-temperature thermal conductance of phononic crystals. The thermal properties quantitatively match that calculated from the modified dispersion relation. Reproduced with permission. ${ }^{[18]}$ Copyright 2014, Springer Nature.

\begin{tabular}{|c|c|c|}
\hline Fabrication technique & Technical remarks & Reference \\
\hline \multicolumn{3}{|c|}{ Top-down } \\
\hline $\begin{array}{l}\text { Electron beam lithography } \\
\text { and reactive ion etching }\end{array}$ & $\begin{array}{l}\text { Standard and well optimized method for silicon } \\
\text { processing. } \\
\text { Not suitable for all materials. }\end{array}$ & $\begin{array}{l}{[14,16,30,35,49-} \\
52]\end{array}$ \\
\hline Focused ion beam milling & $\begin{array}{l}\text { Suitable for a wide range of materials. } \\
\text { Induces surface damage and amorphisation. }\end{array}$ & {$[38,39]$} \\
\hline
\end{tabular}




\begin{tabular}{|c|c|c|}
\hline \multicolumn{3}{|c|}{ Bottom-up } \\
\hline BCP self-assembly & $\begin{array}{l}\text { Possibility of nm-size features. } \\
\text { Long range order difficult to achieve }\end{array}$ & {$[45,46]$} \\
\hline $\begin{array}{l}\text { Colloidal crystal self- } \\
\text { assembly }\end{array}$ & $\begin{array}{l}\text { Variety of assembly methods for large area and } \\
\text { highly ordered crystals }\end{array}$ & {$[42]$} \\
\hline
\end{tabular}

Table 1: Summary of suitable techniques for the fabrication of 2D PnCs.

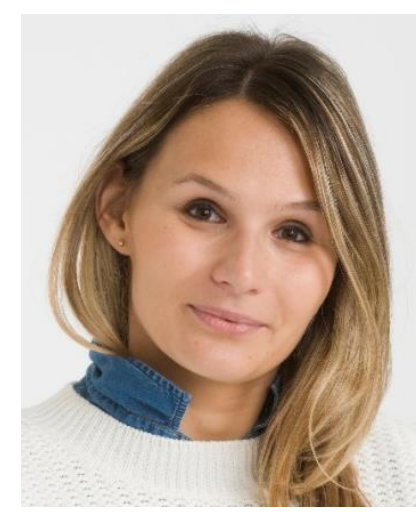

Marianna Sledzinska received her PhD from Autonomous University of Barcelona in 2012. Since then her research is focused on nanofabrication and heat transfer in 2D membranes and phononic crystals.

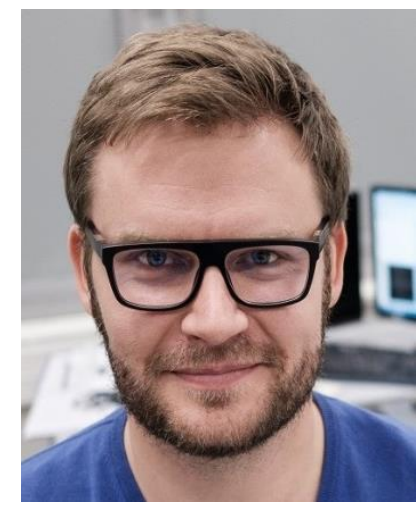

Bartlomiej Graczykowski is a group leader at the Faculty of Physics of Adam Mickiewicz University in Poznan, Poland and guest researcher at the Max Plank Institute for Polymer Research in Mainz, Germany. His current research activities include advanced inelastic light scattering techniques (Brillouin, Raman) applied to nanostructures, hypersound and heat transport at the nanoscale in confined and organized systems. 


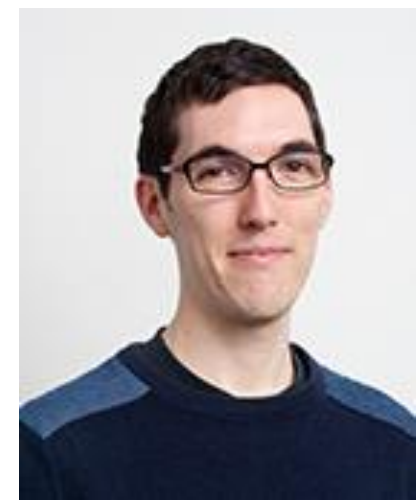

Jeremie Maire is a postdoctoral researcher at the Catalan Institute of Nanoscience and Nanotechnology. He received his Ph.D. from École Centrale de Lyon, in France, after investigating the thermal properties of silicon nanostructures in the University of Tokyo. His current research is shared between the study of optomechanical cavities at room temperature and optical spectroscopy of thermal properties of nanostructures and 2D materials.

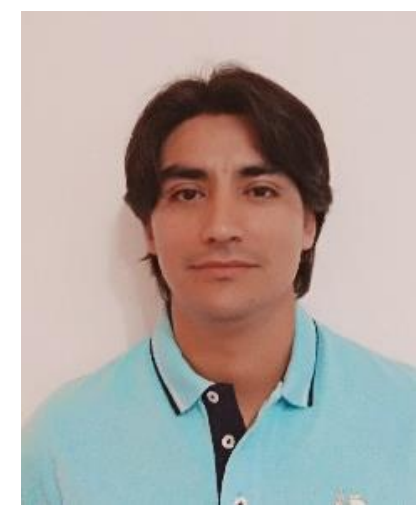

Emigdio Chavez-Angel is a postdoctoral researcher at the Catalan Institute of Nanoscience and Nanotechnology, Barcelona, Spain. He received his Ph.D. from Universitat Àutonoma de Barcelona (UAB) in 2014. He obtained Bachelor and Master in Physics from Universidad Católica del Norte, Antofagasta, Chile in 2008 and 2010, respectively. Currently, his research activities are focused on nanoscale thermal transport in solid systems, nanofluids and molten salts. 


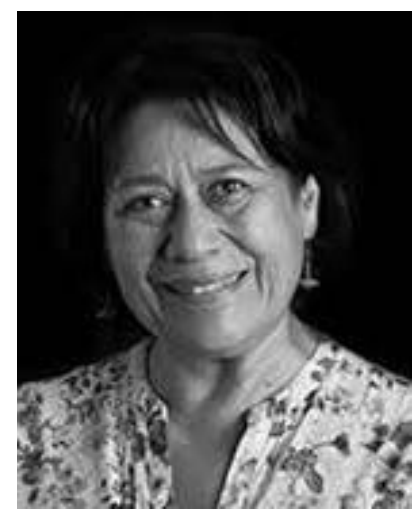

Clivia M. Sotomayor-Torres obtained her PhD in Physics in 1984 from Manchester Univ. (UK). She held tenured academic appointments in the UK (St Andrews and Glasgow), Germany (Wuppertal) and Ireland (Cork). Since May 2007 she is with ICREA based at the Catalan Institute of Nanoscience and Nanotechnology, where she is the leader of the Phononic and Photonic Nanostructures Group. Her main research interests focus on the field of science and engineering of phononic nanostructures, nanophotonics, and novel lithography methods for their realization, such as nanoimprint lithography and self-assembly.

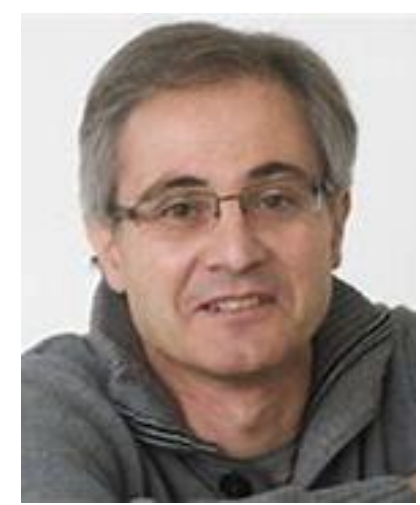

Francesc Alzina is a senior researcher at the Catalan Institute of Nanoscience and Nanotechnology (ICN2). After receiving his $\mathrm{PhD}$ from the Universitat Autonoma de Barcelona, he was appointed as postdoctoral researcher at the National Renewable Energy Laboratory (NREL) in USA and, later, at the Paul Drude Institute in Germany. He joined the Phononic and Photonic Nanostructures Group in 2011 and, since then, he has been performing research on the phonon-related properties of low dimensional and nanostructured materials, with interests spanning from control of phonon propagation and confinement in phononic crystals and cavities to thermal transport by phonons. 